\title{
COMMENT
}

\section{Reaffirming that every poke counts! Higher repeated pain exposure in early life linked with greater short- and long-lasting alteration of the nociceptive system}

\author{
Marsha L. Campbell-Yeo (iD ${ }^{1,2}$ \\ Pediatric Research (2020) 87:15-16; https://doi.org/10.1038/s41390-019-0565-7
}

Over the past three decades, researchers have attempted to address the optimal treatment of recurrent pain exposure in vulnerable, prematurely delivered infants requiring specialized neonatal care. Emphasis has been placed on eliminating the myth that preterm infants do not feel or remember pain, accurate assessment of pain in nonverbal populations, consequences of untreated pain, optimal management, underlying mechanisms associated with pain-relieving interventions, and, more recently, novel knowledge translation strategies targeting practice change.

One concerning element raised by this body of pain research relates to the frequency of repeated untreated exposure to tissuebreaking procedural pain in this high-risk population. A review of epidemiological studies reported 7-17 exposures daily, with less than half associated with any form of pain relief. ${ }^{1}$ Moreover, this untreated pain in early life has been linked with immediate adverse physiological effects as well as long-lasting alteration in pain sensitivity and response, brain development, growth, cognitive function, behavioral concerns, emotional regulation, and epigenetic changes. ${ }^{2}$

In their paper titled "Repetitive noxious stimuli during early development affect acute and long-term mechanical sensitivity in rats" van den Hoogen and colleagues further elucidate the shortand long-term effects of repeated pain exposure in early life. Their team aimed to quantify variation of exposure (4 versus 10 procedures per day in 79 Sprague-Dawley male and female rat pups who received either four or ten tactile stimulations (cotton swab to the left hind paw), or four or ten noxious needle pricks from P0 to P7), and investigate the impact the number of repetitive painful stimuli during early development on the maturation of the nociceptive system. ${ }^{3}$ Outcomes included behavioral sensitivity assessed during the neonatal period and adulthood, and after re-injury of the same dermatome in adulthood.

The findings support that exposure to repeated tissuebreaking events leads to altered mechanical sensitivity in both the early postnatal period and into adulthood, but does not appear to effect thermal sensitivity. Additionally, an increased number of neonatal repetitive needle prick exposures in early life heightened ipsilateral mechanical sensitivity in the early postnatal period and mechanical hyposensitivity in adulthood. While higher pain exposure was not associated with altered recovery of mechanical hypersensitivity after ipsilateral paw incision in adulthood, any exposure to early pain was associated with increased postoperative hypersensitivity compared to the touch controls. No differences between sex of the pups were found.

Strengths of this study included the use of littermates and equalization of time away from the dam by removing the entire nest during each procedure. The consistency of many of the findings across previous studies provides additional support of the importance of reduction of exposure of procedural pain in early life for infants delivered preterm. ${ }^{4}$

Despite these strengths, there are some limitations that merit discussion, many addressed by the authors. As described previously and acknowledged by the authors, the use of thermal testing may not be the most relevant outcome of interest when attempting to inform human neonatal populations. Given the emerging human data related to altered brain development and association with cognitive and emotional outcomes, greater emphasis on outcomes related to brain morphology, learning, anxiety, and social behaviors may be more relevant and of greater interest.

Another area of note in the rat model compared to the neonatal intensive care unit (NICU) environment is that rat pups were separated very briefly from the dams, while preterm infants could experience many days and even months isolated from their mothers. As such, the point raised by the authors related to maternal separation and possible link to hyposensitivity are of interest to the NICU context. Additional research examining effects of maternal separation both in pain and non-pain contexts are warranted and highly relevant both with respect to degree of effect but also, and perhaps more importantly, the potential for greater maternal contact to mitigate adverse outcomes, in keeping with previous work supporting this hypothesis. ${ }^{5}$

The authors conclude that increased efforts to reduce pain in early life as well as optimally treating pain should be undertaken in NICUs. The findings of this paper support that higher exposure to pain in early life is associated with greater risk for both shortand long-term adverse effects. To accomplish the goal of reduced pain, there remain two areas of focus. The first would be targeted efforts to eliminate unnecessary procedures and the second to ensure the consistent use of optimal pain reducing interventions.

While we have seen some progress in reducing pain exposure, large variations across neonatal units indicate that considerable improvements could be made to reduce exposure. ${ }^{1}$ Rather than providing a one size fits all approach, utilization of a more individualized risk benefit treatment plan is one of the most effective ways to reduce exposure pain. For example, currently,

\footnotetext{
${ }^{1}$ School of Nursing, Dalhousie University, Halifax, NS, Canada and ${ }^{2}$ Centre for Pediatric Pain Research, IWK Health Centre, Halifax, NS, Canada
}

Correspondence: Marsha L. Campbell-Yeo (Marsha.Campbell-Yeo@Dal.ca)

Received: 8 July 2019 Accepted: 17 August 2019

Published online: 6 September 2019 
the majority of low birth weight infants (those $<1500 \mathrm{~g}$ at birth) undergo blood collections and often lumbar punctures to evaluate for the presence of infection and receive long courses of antibiotic therapy requiring intravenous therapy, regardless of the degree of risk. Incorporating antibiotic stewardship practices targeting atrisk neonates is an excellent example of a pain reducing individualized approach. Similarity the routine measurement of weekly hemoglobin or nutritional bloodwork, or "routine" every 1-3 hourly suctioning versus "as needed" are oblivious areas that could be targeted.

With respect to best practice, there remain many unanswered questions related to the optimal treatment of tissue-breaking and non-tissue-breaking procedures in this population, especially for moderate-to-severe pain-inducing treatments such as retinopathy of prematurity examinations. ${ }^{6}$ Lastly and perhaps most importantly, it is important to note that the bulk of research examining repeated pain exposure in early life has focused on consequences of untreated pain. There is an urgent need to place emphasis on better understanding which pain-relieving interventions would potentially mitigate adverse consequences. It is likely that models targeting the potential protective nature of maternal care have the greatest potential.

In summary, there is mounting evidence regarding the association between early repeated untreated pain, independent of other factors associated with prematurity, with the work of van Hoogen and others demonstrating that these effects are likely cumulative. ${ }^{3}$ From a clinical perspective, emphasis on novel and effective knowledge translation strategies and greater parent involvement are imperative to create the practice change needed to reduce pain exposure. Despite required efforts to reduce pain exposure, there remain numerous life-saving procedures that many of these tiny patients must endure. Practice policies ensuring the most highly skilled care providers conduct these procedures is another way to mitigate repeated exposure as well as ensuring that effective pain-reducing interventions are provided during all exposure. Going forward, researchers should place greater emphasis on determining optimal ways to mitigate the adverse effects of pain exposure. This would be best done through greater integration of basic and clinical science collaborations in which animal models may better control for the many potential confounding factors of the NICU environment. Finally, it remains for future studies to evaluate to what extent brain alterations associated with neonatal pain exposure are related to cognitive, motor, and behavioral outcomes in these infants.

\section{AUTHOR CONTRIBUTIONS}

M.L.C.-Y. is responsible for all contributions to article conception, drafting, and revising of the article, as well as approval of final version.

\section{ADDITIONAL INFORMATION}

Competing interests: The author declares no competing interests.

Publisher's note Springer Nature remains neutral with regard to jurisdictional claims in published maps and institutional affiliations.

\section{REFERENCES}

1. Cruz, M. D., Fernandes, A. M. \& Oliveira, C. R. Epidemiology of painful procedures performed in neonates: a systematic review of observational studies. Eur. J. Pain. [Internet] 20, 489-498 (2016).

2. Walker, S. M. Long-term effects of neonatal pain. Semin. Fetal Neonatal Med. [Internet] https://doi.org/10.1016/j.siny.2019.04.005 (2019).

3. van den Hoogen, N. J., Patijn, J., Tibboel, D. \& Joosten, E. A. Repetitive noxious stimuli during early development affect acute and long-term mechanical sensitivity in rats. Pediatr. Res. (2019) https://doi.org/10.1038/s41390-019-0420-x [Epub ahead of print].

4. Gokulu, G. et al. Comparative heel stick study showed that newborn infants who had undergone repeated painful procedures showed increased short-term pain responses. Acta Paediatr. [Internet] 105, e520-e525 (2016).

5. Mooney-Leber, S. M., Spielmann, S. S. \& Brummelte, S. Repetitive neonatal pain and reduced maternal care alter brain neurochemistry. Dev. Psychobiol. [Internet] 60, 963-974 (2018).

6. Disher, T., Cameron, C., Mitra, S., Cathcart, K. \& Campbell-Yeo M. Pain-relieving interventions for retinopathy of prematurity: a meta-analysis. Pediatrics 142, https://doi.org/10.1542/peds.2018-0401 (2018). 